Relations industrielles

Industrial Relations

\title{
Toward a Theory of Wage Structure, L.R. Salkever, Humanities Press, Inc., New York. 1964, 161 pages.
}

\section{Fernand Blais}

Volume 21, numéro 1, 1966

URI : https://id.erudit.org/iderudit/027654ar

DOI : https://doi.org/10.7202/027654ar

Aller au sommaire du numéro

Éditeur(s)

Département des relations industrielles de l'Université Laval

ISSN

0034-379X (imprimé)

1703-8138 (numérique)

Découvrir la revue

Citer ce compte rendu

Blais, F. (1966). Compte rendu de [Toward a Theory of Wage Structure, L.R.

Salkever, Humanities Press, Inc., New York. 1964, 161 pages.] Relations

industrielles / Industrial Relations, 21(1), 105-105.

https://doi.org/10.7202/027654ar

Tous droits réservés (C) Département des relations industrielles de l'Université Laval, 1966
Ce document est protégé par la loi sur le droit d'auteur. L’utilisation des services d'Érudit (y compris la reproduction) est assujettie à sa politique d'utilisation que vous pouvez consulter en ligne.

https://apropos.erudit.org/fr/usagers/politique-dutilisation/ 
tels ceux de l'administration, de la vente, de la production, des services et de la recherche.

Une deuxième section contient une liste des entreprises et sociétés qui participent à la réalisation de cette documentation, une multitude d'informations concernant ces entreprises, avec des indications sur les genres d'emplois offerts par ces dernières, emplois tant permanents que temporaires. . Ces sociétés participantes sont ensuite classées selon les disciplines sous lesquelles elles offrent du travail ce qui facilite plus encore une recherche rapide des renseignements. Le tout est complété par une brève biographie de presque toutes les compagnies les plus importantes du Canada.

Bref, c'est un instrument de travail des plus pratiques pour celui qui s'occupe de placement ou qui est à la recherche d'un endroit de travail qui lui convienne.

\section{Ronald Pleau}

Toward a Theory of Wage Structure, L.R. Solkever, Humanities Press, Inc., New York. 1964. 161 pages.

Déjà plusieurs volumes traitent de ce sujet, et l'auteur y puise largement. En fait, ce volume n'apporte rien de nouveau en soi. II est un inventaire de ce qui est fait comme l'auteur le signale dans la préface: « a critical evaluation of the more significont contributions of past scholarship is in order $\boldsymbol{n}$.

Ce volume aborde le problème en analysant la situation à partir de lo période pré-classique jusqu'à la période contemporaine, traitant du marginalisme, du subjectivisme et de la négociation collective. De sorte que la critique des différents auteurs sur le sujet permet à Salkever de retracer les éléments d'une théorie de la structure des solaires.

Lo construction de cette théorie veut se faire selon une sorte de continuum ò partir de l'élaboration des premières théories, en établissant un pont entre le passé et le présent. L'auteur cherche un modèle pour expliquer \& the occurence of differences in wage payment » (préfoce) quant ò certains facteurs: le niveau d'habileté, l'occupation, l'industrie, la location géographique, l'urbanisation, le sexe, l'âge, l'identification ethnique et l'affiliation syndicale.

Néanmoins, ce volume présente une perspective historique qui permet d'avoir un résumé des différentes approches déjà faites.

Fernand Blais
Les cadres supérieurs dans l'entreprise, $R$ Allusson, Entreprise moderne d'Edition, Paris. 1965. 141 pages.

L'auteur se propose comme but d'entreprendre une description se rapprochant du besoin que toute entreprise a de codres agissant selon une autorité déléguée du patron. II étudiera donc ici les échelons supérieurs de l'entreprise qu'elle soit de type personnel avec comme exemple le présidentdirecteur ou de type anonyme avec par exemple le conseil d'administration.

Le mérite de cet ouvrage consiste à nous présenter un essai de définition du cadre supérieur autour duquel il existe tellement de confusion. "Quelle est l'autorité d'un tel codre? II est appelé à un poste important, il exerce des fonctions qui engagent l'entreprise, qui sont susceptibles de compromettre l'existence même de celle-ci. Autre point: pendant combien de temps autrement que sur le papier, méritero-t-il effectivement de conserver la qualification de supérieur? Pour faire partie du cerveau de l'entreprise certaines qualités, certains dons sont nécessaires?

Huit chapitres constituent le contenu de cet ouvrage très logiquement élaboré. Après nous être renseignés sur la façon dont il est possible de définir un cadre supérieur d'une manière assez précise et compréhensive (chap. 1), nous découvrons l'importance numérique de ces derniers dans les entreprises françaises (chap. II).

Pour nous donner une idée des résultats auxquels parvient l'auteur, mentionnons la définition de cadre supérieur qu'il propose à ses lecteurs. « Est cadre supérieur toute personne qui, attachée à une entreprise, est appelée par ses fonctions à prendre ou à proposer (si elle n'en a pas le pouvoir définitif) toute décision susceptible d'exercer une action sur l'évolution ou sur l'avenir à long terme de cette entreprise $»$. (p. 17)

Pour bien comprendre cette définition il est ensuite nécessaire d'expliquer en quoi consiste le rôle de l'individu en question (chap. III) et quels sont les caractéristiques des différents postes qu'il peut occuper (chap. IV).

Et pour devenir un cadre vraiment supérieur conscient de son rôle et de ses responsabilités, il est nécesaire d'étendre ses horizons au delà de sa vie professionnelle et de parfaire sa culture (chap. V) en plus de savoir aménager ses activités quotidiennes qui peuvent et même doivent être familiales, sociales, publiques et religieuses (chap. VI). 